\title{
Cheminement des eaux superficielles et télédétection pour la modélisation hydrologique distribuée
}

\author{
Water paths by remote sensing for distributed hydrological modelling \\ CHRISTIAN PUECH, JEAN STÉPHANE BAILLY \\ UMR TETIS, Cemagref Montpellier \\ Maison de la télédétection, 500 rue JF breton, 34093 Montpellier cedex 5 \\ Tel 00.33.4.67.54.87.45, puech@teledetection.fr, bailly@teledetection.fr
}

\section{$L$}

es cheminements superficiels des eaux sont de mieux en mieux décrits à partir des informations spatiales. En particulier les MNT à très haute résolution spatiale apportent des données nouvelles qui autorisent une description fine des réseaux d'écoulement superficiel et donc l'intégration de ces structures dans les modélisations hydrologiques distribuées. Mais si les techniques numériques d'observation ont fait de grands progrès dans les années passées, par contre il n'en est pas de même pour les concepts en modélisation et les expérimentations de terrain, ce qui provoque un point de blocage pour une réelle utilisation des données de télédétection finement résolues dans les modèles. Le développement de nouvelles techniques de mesure sur le terrain, de type low cost et réseaux communicants, est porteur de renouveau dans ce domaine.

$W$

ater paths are better and better described through spatial data. High resolution DTM are a good solution for mapping fine water networks and their integration into hydrological modelling. But if, in past years, big progresses concerned spatial data techniques, on the other hand they did concern concepts in modelling and ground experiments. And this is a difficulty for a real use of remote sensing data into finely resolved models. New possibilities are carrying revival in this field, through development of low cost ground techniques and communicating wireless networks.

\section{INTRODUCTION}

Décrire l'espace à partir de données spatialisées devient de plus en plus aisé, tant au niveau des données - baisse des coûts, augmentation de l'offre - que par la facilité de traitement. La discrétisation spatiale qu'offrent ces données pousse à une hydrologie distribuée où l'impact local des états de surface serait implicite. Mais l'utilisation concrète de ces informations en modélisation hydrologique apparaît très délicate. En effet les concepts ou méthodes relatifs à modélisation distribuée avec de la haute résolution spatiale ne sont pas simples à mettre en œuvre. Les mélanges d'échelles des différentes variables utilisées sont souvent mal appréhendés, mettant de la confusion dans la prise en compte de l'impact des différents items. Parallèlement, l'observation hydrologique in situ reste limitée, rendant les calages des modèles hydrologiques incertains, sur-paramétrés. La facilité est d'utiliser les données en mode agrégé sur le bassin, et l'apport potentiel d'une connaissance fine et spatialisée dans les modèles est alors peu valorisé.

Observer et définir ce drain est difficile, d'autant plus que les questions de processus relatifs aux circulations élémentaires des eaux sur les bassins versants, ainsi que la séparation entre écoulements de surface ou souterrain, sont objets de polémiques.
On met alors en évidence un gap croissant entre d'une part la description de plus en plus précise de l'espace bassin versant et d'autre part la conceptualisation et la modélisation en mode distribué du fonctionnement hydrologique qui restent souvent peu convaincantes car reposant sur des observations locales très peu nombreuses. Ce déséquilibre est source de blocages. En nous focalisant sur la connaissance spatialisée des cheminements des eaux de surface, nous présentons quelques idées sur les avancées actuelles sur ces deux points, la vision purement descriptive, par des instruments aéro-spatiaux, de l'espace hydrologique de plus en plus précise et généralisable, et la vision de son fonctionnement hydrologique, difficile à appréhender à la même résolution.

Notre objet d'étude porte sur les écoulements concentrés de surface (réseaux hydrographiques). Les chemins de l'eau sont essentiels dans les modélisations finement distribuées, et une approche objective des drains et de leur fonctionnement est incontournable si l'on veut améliorer ces modélisations.

Reprenons les deux fonctions de base utilisées en hydrologie : production et transfert. A partir des images de télédétection, la recherche a fait, dans les années passées, de gros efforts centrés surtout sur la fonction de production : travaux sur le rôle de l'occupation du sol, les bilans, le rôle de l'humidité, les modèles énergétiques SVAT, c'est-à-dire très focalisés sur une approche verticale de l'hydrologie à la par- 
celle et moins sur les flux latéraux de surface. La question qui se pose est alors la suivante : la télédétection peut-elle aussi améliorer la connaissance des transferts, des cheminements de l'eau superficielle, de leur dynamique?

L'interrogation suivante porte sur l'effet hydraulique de ce drain et l'introduction de cette connaissance dans la modélisation. Du point de vue modélisation distribuée, le transfert de surface est essentiellement lié aux questions de vitesse des eaux, d'échange entre mailles. On souhaite mieux séparer les eaux déjà bien canalisées, où les vitesses sont fortes, en mètres par seconde, même dans les drains élémentaires, et les écoulements en versant où les vitesses de l'eau sont beaucoup plus faibles et dépendent des processus locaux, tels que infiltration, circulation dans les nappes... Dans ce cas on parlera de $\mathrm{mm} / \mathrm{s}$ ou de $\mathrm{cm} / \mathrm{s}$, rarement de $\mathrm{dm} / \mathrm{s}$ sauf si des lignes de circulation préférentielles bien connectées se mettent en eau. La réponse hydrologique du bassin est dépendante de la présence ou absence de ces drains avec un fort impact dans la forme des hydrogrammes : la connaissance fine du réseau de drainage et des chemins de l'eau semble un élément important dans une modélisation cohérente du transfert des eaux.

Les drains sont les éléments clefs des changements de circulations d'eau sur les bassins versants : le passage du versant aux drains constitue en effet une rupture entre les circulations de surface ou de nappe (effets surfaciques) et les circulations canalisées (effets linéaires). La présence d'un drain en eau augmente l'efficacité de transport du débit vers l'aval, les circulations des masses d'eau deviennent différentes, la réponse du bassin est modifiée : les processus hydrologiques élémentaires associés sont alors importants à observer et à modéliser. Mais pour que ces informations soient utilisables en modélisation spatialisée l'observation doit aussi fournir une vue spatialisée du phénomène.

\section{CHEMINEMENTS DES EAUX : RÉSEAU POTENTIEL, RÉSEAU RÉEL}

Repérer les drains et les introduire dans une modélisation nécessite une réflexion préalable sur la définition même du réseau de drainage. Comment le définir ? Comment le cartographier et quel réseau hydrographique est utile pour la modélisation? Nous proposons de simplifier le problème en considérant deux types de réseaux : celui des formes du relief (drains secs ou potentiels) et celui avec présence avérée de circulations d'eau (drains en eau ou réels), associé sur le terrain aux questions d'humidité des sols, de présence de ripisylve, de forêt galerie, ...

Le réseau potentiel de drainage est relatif aux formes $\mathrm{du}$ terrain naturel, vallons, courbures ... C'est la forme du relief qui le caractérise, défini par les formes concaves ou convexes du terrain. Il est observable par imagerie spatiale par exemple grâce aux ombrages, mais il est plus généralement observé sur MNT. Il est défini indépendamment de son état hydrologique, comme potentiel de transport des eaux. Sa détection et sa cartographie ne sont pas absolues, elles dépendent de la précision d'observation, mais, globalement, ce réseau de drains est constant, une fois que l'on a déterminé la méthode d'observation.
Le réseau réel de drainage ou drain en eau est relatif au fonctionnement hydrologique et aux circulations d'eau. Il comprend souvent les « traits bleus » des cartes IGN dans les zones aval du bassin. Toutefois dans la partie amont du réseau, là où s'effectue la genèse des crues, ce drain en eau est essentiellement variable au cours du temps. Son observation sera donc délicate, variable. Le tracé du drain en eau sera a priori plus restreint que celui du drain potentiel.

Le problème de détection est alors ramené aux questions de l'observation de ces deux types de drains à partir des données spatiales.

\section{III — LA DESCRIPTION DES RÉSEAUX POTENTIELS À PARTIR DES DONNÉES SPATIALISÉES}

Dans la description fine des chemins des eaux de surface par télédétection où en est-on aujourd'hui ?

\section{III.1 À PARTIR DES MNT}

Les MNT à 50 ou $90 \mathrm{~m}$ sont complétés aujourd'hui par des relevés plus précis : images satellites métriques ou encore relevés aéroportés par exemple par Lidar qui conduit fréquemment à des résolutions métriques avec une incertitude décimétrique sur l'altitude. Enfin signalons les moyens aéroportés proches (ULM, Drones...) dont les prises de vue stéréoscopiques peuvent générer des MNT avec des résolutions au sol centimétriques [1].

Les techniques d'extraction de réseaux à partir de MNT sont aujourd'hui classiques [2]. Elles utilisent la plupart du temps des algorithmes raster avec détection des circulations arborescentes, après avoir éliminé les incohérences hydrauliques du produit initial.

Ces réseaux sont cohérents sauf dans les zones peu variables - zones planes ou versants réguliers - où il faut compléter l'information avec des données extérieures (ex le Stream Burning) et dans l'amont des bassins par la difficulté d'arrêter le départ de drains au bon endroit : "Where do Channel Begin ? » [3] (Montgomery \& Dietrich, 1988). En opposition aux solutions raster, des solutions à partir de format TIN se sont développées pour mieux introduire les contraintes anthropiques ou mieux définir les cheminements à l'amont des bassins ([4]; [5]).

En théorie, à partir d'un MNT on peut déterminer un réseau de drainage passant par tous les points de l'espace, jusqu'au point le plus amont - pixel ou triangle. En pratique, on ne doit pas remonter le réseau au-delà d'une certaine limite, sous peine de voir les cheminements calculés non conformes à la réalité du terrain. Les solutions classiques arrêtent le tracé sur un critère uniforme de surface amont drainée, parfois modulés en fonction de données externes telles que l'occupation du sol ou la géologie. Ces résultats sont localement incohérents, d'où l'émergence forte actuellement de critères d'arrêt basés sur la courbure locale. Selon cette idée on dessinera le réseau tant que le MNT indique la présence locale de vallons ou ravines, en se basant sur la courbure locale du terrain naturel ou celle 

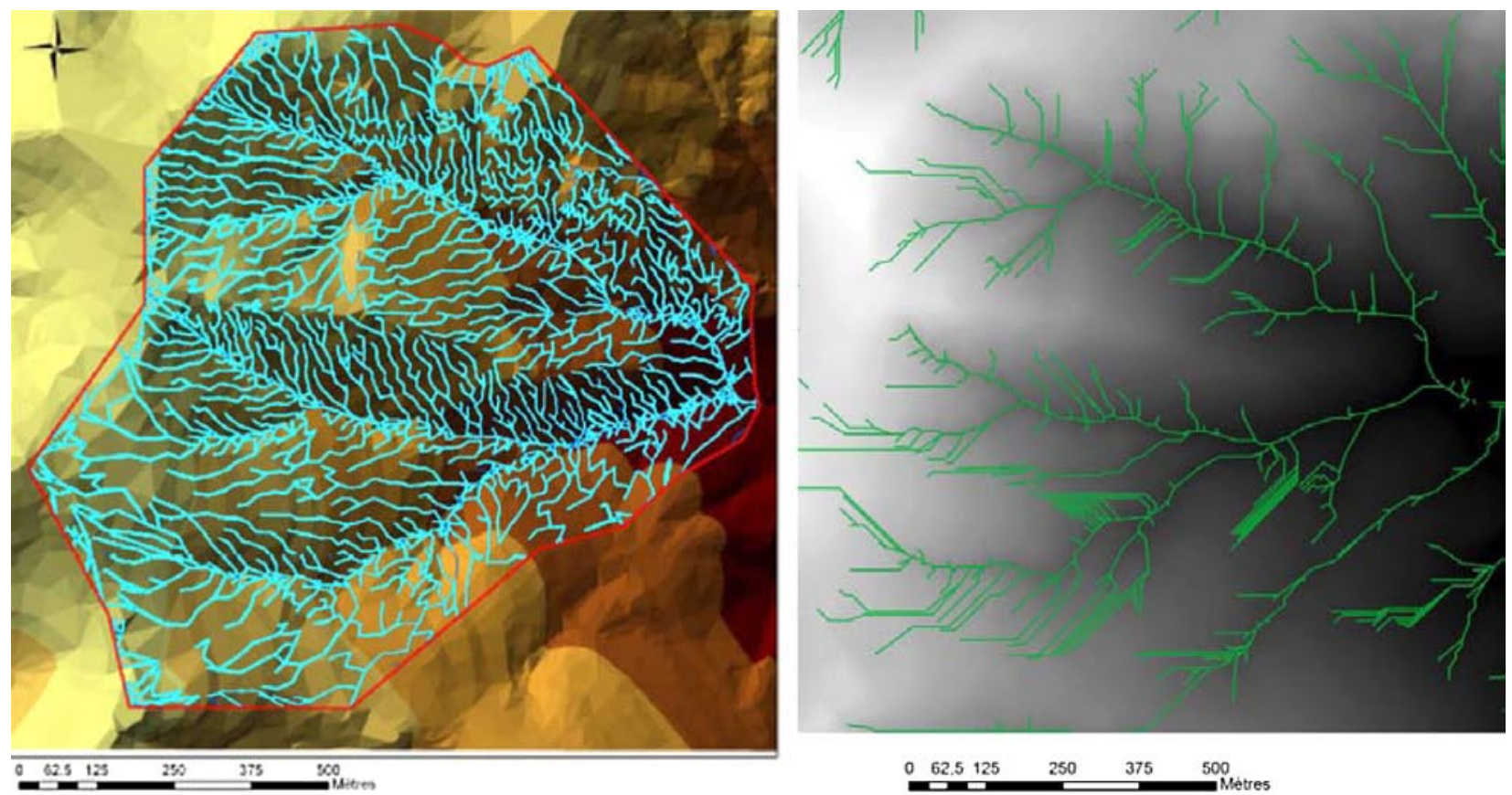

Figure 1. Alternatives TIN ou raster pour calculer les réseaux a l'amont des bassins. Test sur le bassin de Tourgueille, Cévennes $\left(1 \mathrm{~km}^{2}\right)$.

des lignes de niveau. Le réseau final est dépendant de la résolution du MNT.

Avec les lidars aéroportés, les résolutions spatiales sont de l'ordre du mètre. Dans ces conditions, la précision du modelé de terrain fait passer une étape dans l'amélioration de la description. Ainsi les routes et infrastructures sont explicitement prises en compte dans le MNT lidar (figure 2), quand il fallait auparavant rectifier les MNT plus grossiers en incrustant ces infrastructures [6]. Ceci est donc directement intéressant par exemple dans l'hydrologie de montagne où l'on sait que pistes et ouvrages anthropiques sont autant de cheminements importants qui influent dans la genèse des crues.
Dans les cas de résolution fine, les objets sont facilement détectables quelque soit l'algorithme utilisé ; choisir le meilleur algorithme semble alors peu déterminant pour détecter les éléments. Ainsi les MNT métriques peuvent définir des objets anthropiques tels que les routes bien détectées même avec les algorithmes les moins performants [7]. Au contraire, le problème du choix d'un algorithme efficace reste important avec des résolutions plus grossières. Pour généraliser, nous pouvons associer la finesse du MNT (et donc son potentiel de détection d'objets) au rapport adimensionnel $\mathrm{o} / \mathrm{r}$ (o taille de l'objet analysé et $\mathrm{r}$ résolution du MNT) : quand o/r est supérieur à 3 environ on considèrera que les MNT sont très fins.
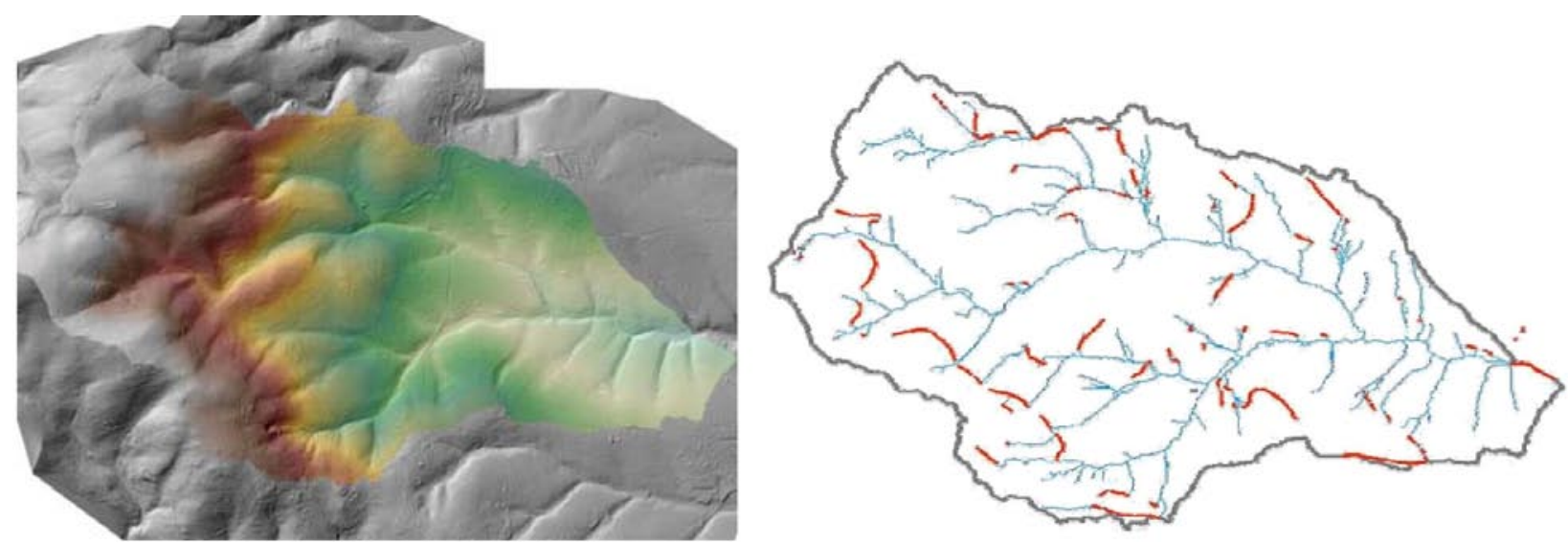

Figure 2. Un MNT à $2 \mathrm{~m}$ de résolution issu de Lidar aéroporté ; Bassin de l'Yseron (69). La figure de droite met en évidence les éléments anthropiques (routes et chemins) présents dans le MNT Lidar et conduisant à des circulations d'eau modifiées [7]. 


\section{III.2 À PARTIR DES IMAGES SATELLITES}

La détection des drains est plus complexe sur images qu'avec le MNT. Sur image la détection est différente. On y repère peu ou mal les formes naturelles (vallons, dépressions naturelles). La détection va concerner une recherche de linéaires, ruptures radiométriques ou alignements, qui sont révélateurs tantôt de formes (drain sec) tantôt d'écoulements (drains en eau), ce qui rend plus difficile la séparation entre ces deux groupes. Le traitement d'image, par classification ou autre méthode, peut conduire à des zones (groupes de pixels) plus ou moins larges et souvent discontinues quand, pour notre schématisation des drains, il est souhaitable d'obtenir une arborescence linéaire. Il faudra donc à un certain moment passer des surfaces aux lignes, puis connecter ces lignes entre elles pour en faire une arborescence continue.

La détection est donc mixte : recherche de formes et recherche de surfaces ou de linéaires révélateurs de présence d'eau, sachant que l'eau libre est peu visible sur images, surtout en zones amont. Formes ou présence habituelle d'eau sont détectables indirectement selon plusieurs indices :

- éclairements différentiels de versants en zone de montagne.

- présence d'une ripisylve forte ; dans les régions semi arides, le tracé des rivières est visible même sur imagerie à 20 ou $30 \mathrm{~m}$ de résolution.

- oppositions de végétation ou d'occupation du sol entre rives.

Dans le cas des Cévennes avec des images Quickbird (image de février 2007, projet ORFEO-CNES), une extraction manuelle des fonds de talwegs a été effectuée (figure 3).
Comparée aux traits bleus des cartes IGN, cette extraction est apparue plus fine et plus riche. Mais on s'aperçoit rapidement de la non-isotropie de détection des ravines. En versant nord la qualité de détection est globalement très bonne. En versant sud, face au soleil, la détection est plus limitée [8].

Les cartographies obtenues à partir de ces images ne sont donc pas toujours très homogènes et donc difficilement utilisables en modélisation. Par contre leur intérêt peut venir en appui des extractions de drain sur MNT, comme référence « de terrain » pour définir où arrêter les drains : la ripisylve ou la végétation renforcée le long des rives indique une présence d'eau régulière et donc un drain possible.

Les problèmes d'échelle spatio-temporelle compliquent ces détections. Ainsi espérer arriver à une représentation complète du réseau hydrographique à partir d'imagerie satellite dans ce type de contexte apparaît totalement illusoire. Nous illustrons ce type de difficulté dans le cas de l'échelle spatiale à travers la figure 4. Elle présente côte à côte la photo d'un éclair un jour d'orage et un dessin de réseau hydrographique correspondant à un de nos bassins cévenols (Peyrolles, $4 \mathrm{~km}^{2}$ ). La similitude générale est frappante audelà des différences de schématisation. La vision photographique de l'éclair révèle de multiples branches, certaines très fines, peu visibles. Le détail de la ramification de l'éclair montre la difficulté de proposer une mesure unique de la longueur totale, du nombre de branches, car toute mesure nécessite une schématisation et un passage par des seuils de représentativité, des critères de détection. Au contraire le réseau hydrographique est un dessin schématique obtenu après extraction manuelle; il donne une représentation nette, figée. On imagine aisément que cette représentation consti-
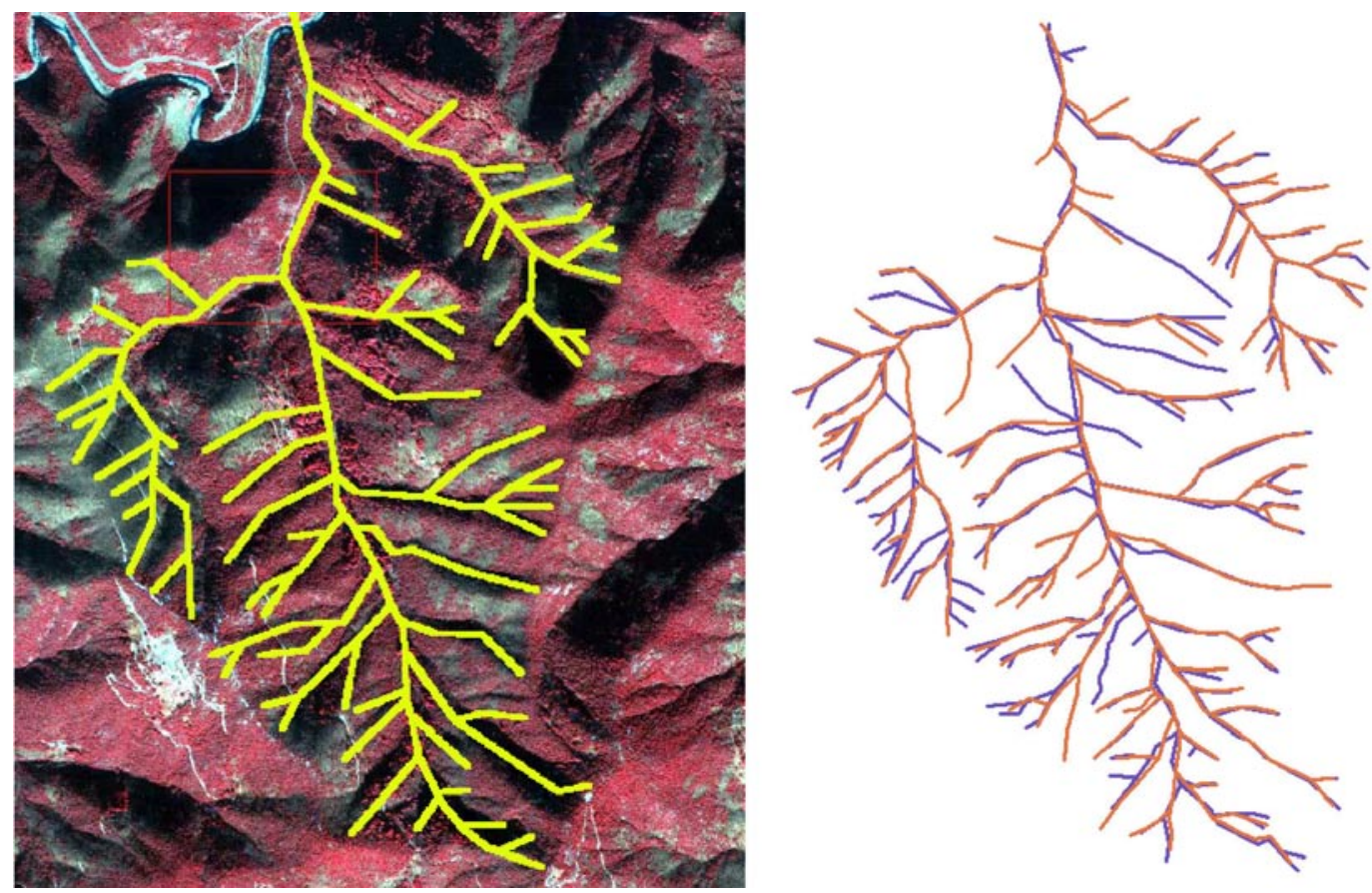

Figure 3. Extraction de réseau hydrographique sur image Quickbird des Cévennes et comparaison au réseau IGN, projet ORFEO-CNES, bassin de Peyrollles $\left(4 \mathrm{~km}^{2}\right)$, [8]. 

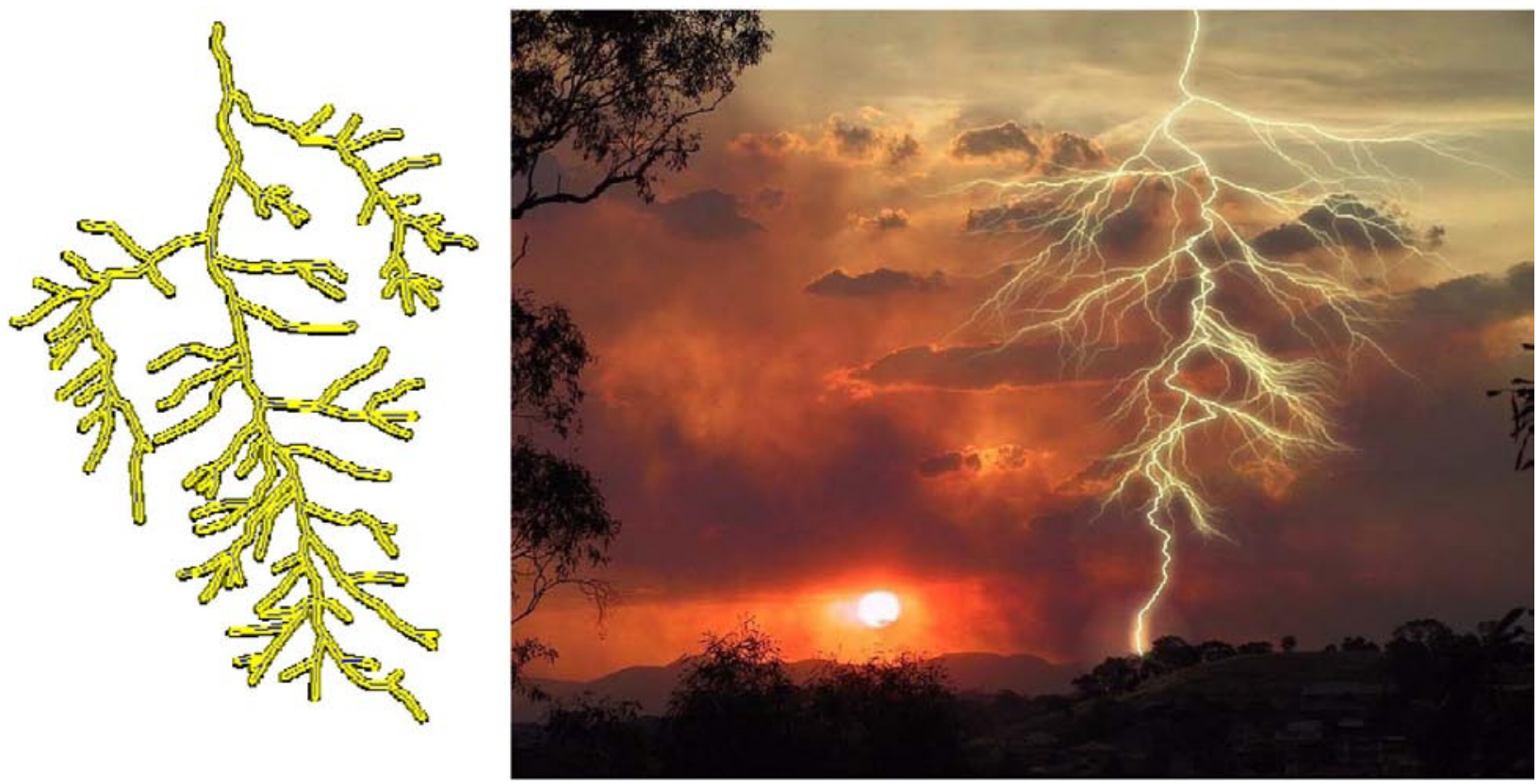

Figure 4. Comparaison visuelle entre un schéma de réseau hydrographique en Cévennes et une photographie d'éclair pendant un orage (fond d'écran gratuit, téléchargeable sur web), mettant en évidence la difficulté d'une schématisation unique.

tue une solution particulière, incomplète et dépendante de l'échelle de travail et des seuils de perception.

\section{IV — LE DRAIN EN EAU, VERS UNE OBSERVATION HYDROLOGIQUE SPATIALISÉE, ASSOCIÉES AUX IMAGES}

Le drain en eau - ou drain réel - est celui qui est en eau à un instant donné, mais aussi celui que l'on souhaite introduire comme composante de compréhension des modèles. Or observer et caractériser ce drain est plus complexe. A priori, on l'a déjà dit, ce sera un sous ensemble variable du schéma de drain potentiel déjà défini.

Dans notre façon de voir les choses il y a plusieurs interrogations de plus en plus complexes : qu'est ce qu'un drain en eau ? Comment le caractériser ? Quel est l'effet sur les circulations ? Quel est le réseau à introduire dans un modèle ?

Le premier point est de définition. Pour nous, ce drain en eau étant celui qui coule, ceci implique que, à l'amont des bassins, il ne peut être stable dans le temps : selon les saisons, ou au cours des épisodes pluvieux, il y a assèchements ou mises en eau successives des drains, que l'on peut décrire comme une « respiration» des drains pendant la période d'observation.

Ce qui nous semble important à noter est que la réponse du bassin à un événement pluvieux sera dépendante non seulement de la modification de l'humidité des sols - et donc de la production locale - mais aussi de la mise en eau d'axes de drainage, susceptibles d'emmener l'eau rapidement à l'aval. Dans ces conditions le réseau de drainage, supposé jusqu'ici implicitement invariable et structurant les modélisations, peut devenir une variable d'ajustement du modèle à la réalité.

Suivre la respiration des drains est une opération très lourde et difficile. Une observation a été réalisée dans les Cévennes dans le sous bassin de Tougueille $\left(1 \mathrm{~km}^{2}\right)$. Une quarantaine de points de mesure ont été définis et observés en des journées clefs au cours de l'année hydrologique 2005-2006. Après une forte pluie d'automne et surtout après un événement pluie et neige sur le Mont Aigoual en début février $2006(200 \mathrm{~mm})$, on a observé la mise en circulation d'un grand linéaire de ravines normalement à sec. Au printemps il y a eu régression progressive du nombre de ravines en eau en lien avec l'assèchement progressif des nappes. L'estimation des longueurs en eau varie ainsi dans un facteur 3 au cours de cette période. Cette observation a pu être réalisée de façon simple, hors périodes de crues, en phases de récession des eaux. Chaque mesure manuelle, sur une quarantaine de points, a été effectuée au cours de la même journée, ce qui permettait de supposer l'instantanéité de l'observation des débits et des linéaires en eau : en $8 \mathrm{~h}$ de récession les changements étaient minimes [9].

En phase de crue, le synchronisme des mesures est beaucoup plus délicat et on ne peut plus se contenter de mesures manuelles en mode multi sites. Mesurer 40 points à la fois par des mesures manuelles est impensable : l'acquisition doit utiliser un système automatique à coût acceptable. Aussi est il nécessaire de développer des modes de mesures simples, légers et à bas prix.

Ceci constitue une nouvelle tendance et un nouveau défi de mesure en hydrologie : effectuer des mesures distribuées dans l'espace et à prix raisonnable. Pour ce qui à trait aux pluies, des solutions opérationnelles existent, notamment à travers 
les réseaux de radar pluies, au sol, par exemple le réseau Aramis, mais avec des résolutions de $1 \mathrm{~km}^{2}$. D'autres variables sont aussi en train d'être auscultées ; mesures de thermométrie ou d'humidité spatialisée, mesure de débits ou de simple présence d'eau. Des observations low cost associées à des réseaux communicants sans fil sont en développement ([10] ; [11]) et vont permettre d'observer spatialement non seulement les formes, le relief, l'occupation des sols, mais aussi les variables du fonctionnement hydrologique (température, humidité, débit, pluie ... etc.). Cette phase est indispensable pour développer une valorisation de la connaissance spatiale, pour développer l'intégration de données images dans les modèles et, finalement, retrouver un certain équilibre entre outils de description spatiale et connaissance des variables in situ. Tant que les observations in situ seront pauvres on aura tendance à générer des modèles sur-paramétrés, qui engendrent de l'équifinalité, donc une difficulté de bien coller au sens physique. Le développement des observations low cost in situ apparaît alors comme un bon outil de connaissance complémentaire apte à valoriser l'utilisation des techniques spatiales sur le bassin et comme point de départ de modélisations distribuées physiquement cohérentes.

\section{$V \square$ CONCLUSION}

Le développement des techniques d'observation spatiale, notamment à très haute résolution, pousse vers des modélisations hydrologiques de type distribué. Cependant si l'observation spatiale prend de plus en plus d'importante et de précision, l'observation in situ et le développement des concepts relatifs à de vraies modélisations distribuées est lent. Les questions d'échelle deviennent importantes, décrire et modéliser l'anthropisme apparaît comme de plus en plus nécessaire quand la résolution de calcul s'affine. Ici, en nous basant sur l'exemple de la description de la structuration des chemins de l'eau par réseau hydrographique, on montre que les données spatiales de plus en plus précises améliorent la connaissance des réseaux potentiels (drains secs) mais que le déséquilibre se creuse avec les observations et les concepts hydrologiques qui restent assez pauvres ; dès lors la mise en œuvre de techniques d'observation in situ, nombreuses et distribuées, multi sites, par techniques low cost, se révèlent être une réponse possible pour une mise en œuvre cohérente de modélisations distribuées, impliquant un meilleur équili- bre entre connaissances spatiales et hydrologiques et réduisant la sur-paramétrisation des modèles.

\section{BIBLIOGRAPHIE}

[1] Jacome A., Puech C., Raclot D., Bailly J. S., Roux B. (2008) - Extraction d'un modèle numérique de terrain à partir de photographies par drone. Revue des Nouvelles Technologies de l'Information, Cépaduès éditions. E-13 79-99

[2] O'Callaghan J., Mark D. (1984) - The extraction of drainage networks from digital elevation data. Computer vision, graphics and image processing. 28 323-344

[3] Montgomery D. R., Dietrich W. E. (1988) - Where do channels begin ?. Nature. 336 232-234

[4] Bocher E. (2005) - Impacts des activités humaines sur le parcours des écoulements de surface dans un bassin versant bocager : essai de modélisation spatiale. Thèse de doctorat en géographie, Université de Rennes $2.367 \mathrm{p}$

[5] Tortrat F. (2005) - Modélisation orientée décision des processus de transfert par ruissellement et subsurface des herbicides dans les bassins versants agricoles. Thèse ENSA de Rennes, UMR INRA-Agrocampus : Sol Agronomie Spatialisation Rennes-Quimper. $173 \mathrm{p}$

[6] Rousseaux F. (2004) - Étude de la cohérence entre données altimétriques et planimétriques dans un contexte de risque. Thèse Université de Marne la Vallée. $160 \mathrm{p}$

[7] SARRAZIN B. (2008) - Exploitation de données hydrologiques à différentes échelles sur le bassin de l'Yzeron. Séminaire AVUPUR, Lyon 12/2008.

[8] Gille J. (2007) - Du drain potentiel au drain réel. Caractérisation spatiale des chemins de l'eau de la Vallée Obscure (Gardon d'Anduze). Master 2 Recherche Eau et Environnement, Univ. Montpellier II. 84 p

[9] Alayoubi M. (2006) - Représentation multi-echelle d'un bassin versant. Application au Bassin de Tourgueille. Mémoire d'ingénieur, ESGT, Le Mans. 70 p

[10] Smith P., Hughes D., Beven K., Coulson G., Blair G. (2007) - On the use of Adaptive Grid-Enabled Wireless Sensor Networks in data collection. EGU EGU2007-A-09510 Wien 2007.

[11] Bogena H., Huisman J. A., Oberdörster C., Vereecken H. (2007) - Evaluation of a low-cost water content sensor for wireless network applications. EGU2007-A-01916 ; HS331M040-002.

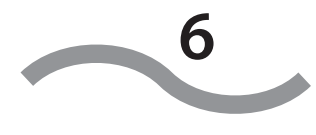

